\title{
Hovenia dulcis Thunb. and its active compound ampelopsin inhibit angiogenesis through suppression of VEGFR2 signaling and HIF-1 $\alpha$ expression
}

\author{
JANG MI HAN, HAET NIM LIM and HYE JIN JUNG \\ Department of BT-Convergent Pharmaceutical Engineering, Sun Moon University, \\ Tangjeong-myeon, Asan-si, Chungnam 336-708, Republic of Korea
}

Received June 26, 2017; Accepted October 3, 2017

DOI: $10.3892 /$ or.2017.6021

\begin{abstract}
Specific inhibition of angiogenesis has been considered a powerful strategy for the treatment of cancer and other angiogenesis-related human diseases. Hovenia dulcis Thunb., Japanese raisin tree or Oriental raisin tree, is a hardy tree found in Asia, Eastern China and Korea and has been known to possess various biological activities, including antifatigue, antidiabetic, neuroprotective and hepatoprotective activity. In the present study, for the first time, we evaluated whether a $100 \%$ ethanol extract of Hovenia dulcis Thunb. (HDT) inhibits the angiogenesis of human umbilical vein endothelial cells (HUVECs) using in vitro angiogenesis assays. HDT suppressed vascular endothelial growth factor (VEGF)-induced proliferation, migration, invasion and tube formation of HUVECs at subtoxic doses. In addition, HDT significantly inhibited in vivo angiogenesis of the chorioallantoic membrane from growing chick embryos without exhibiting cytotoxicity. Furthermore, HDT downregulated not only VEGF receptor 2 (VEGFR2) signaling in HUVECs, but also hypoxia-inducible factor (HIF)-1 $\alpha$ expression in hepatocarcinoma cell line HepG2. Ampelopsin is a bioactive flavanonol found in Hovenia dulcis Thunb. Our data showed that ampelopsin inhibited angiogenesis with no cytotoxicity by suppressing both VEGFR2 signaling and HIF-1 $\alpha$ expression. These results suggest that Hovenia dulcis Thunb. and its active compound ampelopsin exhibit potent antiangiogenic activities and therefore could be valuable for the prevention and treatment of angiogenesis-related diseases including cancer.
\end{abstract}

Correspondence to: Professor Hye Jin Jung, Department of BT-Convergent PharmaceuticalEngineering, Sun Moon University, 70, Sunmoon-ro 221, Tangjeong-myeon, Asan-si, Chungnam 336-708, Republic of Korea

E-mail: poka96@sunmoon.ac.kr

Key words: Hovenia dulcis Thunb., ampelopsin, angiogenesis, cancer, VEGFR2, HIF-1 $\alpha$

\section{Introduction}

Angiogenesis, the process of new blood vessel formation from pre-existing ones, is essential in physiological processes, including revascularization, wound healing, reproduction and pregnancy (1). In contrast, abnormal angiogenesis results in many pathological diseases, such as tumors, rheumatoid arthritis, psoriasis and vasoproliferative retinopathy (2). Particularly, tumorigenesis depends on angiogenesis for metabolic supplies such as essential nutrients, various growth factors and molecular oxygen (3). Therefore, suppression of angiogenesis presents a promising strategy for cancer treatment and other angiogenesis-related human diseases.

Endothelial cell proliferation, metastasis and tubulogenesis are the major hallmarks of angiogenesis, which are induced by the crucial regulator, vascular endothelial growth factor (VEGF) $(3,4)$. VEGF acts on endothelial cells via the vascular endothelial growth factor receptor (VEGFR) family, among which VEGFR2 is a key mediator of proangiogenic activities induced by VEGF (5). During the process of angiogenesis, VEGFR2 signaling activates many downstream mediators, including signal transducer and activator of transcription (STAT)3, extracellular signal-regulated kinase (ERK) 1/2 and $\mathrm{AKT}$, which promote the proliferation, migration and differentiation of endothelial cells (3-5). In addition, in VEGFR2-mediated cascades, matrix metalloproteinases (MMPs) play a key role in extracellular matrix (ECM) degradation and cyclin D1 is required for progression through the G1 phase of the cell cycle, which are deeply involved in the regulation of endothelial cell survival, migration and invasion $(6,7)$. Thus, inhibition of VEGFR2 signal transduction is considered as a therapeutic strategy for antiangiogenesis.

Hypoxia areas arise as a result of the rapid growth of solid tumors (8). During the process of hypoxia-induced malignant progression, tumors may undergo increased invasive growth, tumor cell spreading and metastasis (9). Cancer cells exposed to hypoxic conditions exhibit altered protein levels, such as hypoxia-inducible factor (HIF)- $1 \alpha$ accumulation. HIF-1 $\alpha$ is a crucial transcription factor involved in the angiogenesis in tumor cells, which controls transcription of hypoxia-regulated genes such as VEGF $(10,11)$. Therefore, inhibition of HIF-1 $\alpha$ may be regarded as an antiangiogenic therapy. 
Natural products are rich source of angiogenesis-regulating compounds (12). Some angiogenesis inhibitors have been isolated from natural products, such as paclitaxel (Taxus brevifolia), camptothecin (Camptotheca acuminate), combretastatin (Combretum caffrum) and farnesiferol C (Ferula assafoetida) (13-16). To date, many studies have demonstrated that a variety of natural products have antiangiogenic activities (17). Hovenia dulcis Thunb., Japanese raisin tree or Oriental raisin tree, is a hardy tree found in Asia, Eastern China and Korea, and its roots, seeds, branches, leaves and fruits have been known to possess various biological activities, including antifatigue, antidiabetic, neuroprotective and hepatoprotective activity (18-22). Recently, several studies have revealed that Hovenia dulcis Thunb. extract contains a variety of pharmacological compounds, such as alkaloids, flavonoids and triterpenoids (23). Ampelopsin, also known as dihydromyricetin, is a flavanonol of Hovenia dulcis Thunb. extract and possesses antiinflammatory, antioxidative and anticancer activity (24-26). However, whether Hovenia dulcis Thunb. extract and its active compound ampelopsin inhibit angiogenesis and the underlying mechanisms in endothelial cells are still unknown. In the present study, we investigated the in vitro and in vivo antiangiogenic effects and the molecular mechanisms of Hovenia dulcis Thunb. extract and ampelopsin. Our results demonstrated that Hovenia dulcis Thunb. extract and ampelopsin efficiently suppressed angiogenesis by downregulating both VEGFR2 signaling and HIF-1 $\alpha$ expression.

\section{Materials and methods}

Materials. Ampelopsin was purchased from Sigma-Aldrich (St. Louis, MO, USA) and dissolved in dimethyl sulfoxide (DMSO) at a concentration of $100 \mathrm{mM}$ as a stock solution. Endothelial growth medium-2 (EGM-2) was obtained from Lonza (Walkersville, MD, USA). Dulbecco's modified Eagle's medium(DMEM) and fetal bovine serum(FBS) were purchased from Invitrogen (Grand Island, NY, USA). Recombinant human vascular endothelial growth factor (VEGF), Matrigel and Transwell chamber systems were obtained from Koma Biotech (Seoul, Korea), BD Biosciences (San Jose, CA, USA) and Corning Costar (Acton, MA, USA), respectively. Antihypoxia inducible factor- $1 \alpha(\mathrm{HIF}-1 \alpha)$ antibody was purchased from BD Biosciences. Anti-phospho-VEGFR2, anti-VEGFR2, anti-phospho-STAT3, anti-STAT3, anti-phospho-AKT, antiAKT, anti-phospho-ERK1/2, anti-ERK1/2, cyclin D1, MMP-2, MMP-9 and anti- $\beta$-actin antibodies were purchased from Cell Signaling Technology (Beverly, MA, USA).

Preparation of an ethanol extract of Hovenia dulcis Thunb. The fresh branches of Hovenia dulcis Thunb. (Jangheung, Korea) were fragmented and stored in a closed container. Ethanol extract was obtained by dissolving $150 \mathrm{~g}$ of raw material in 1 liter of $100 \%$ ( $\mathrm{vol} / \mathrm{vol}$ ) ethanol and shaken for $24 \mathrm{~h}$ at room temperature. The mixture was filtered and the clear filtrate was concentrated, vacuum dried and kept at $-20^{\circ} \mathrm{C}$.

Cell culture and hypoxic condition. Human umbilical vein endothelial cells (HUVECs) and HepG2 (human hepatocarcinoma) cells were obtained from the Korean Cell Line Bank (KCLB) and grown in EGM-2 and DMEM supplemented with
$10 \% \mathrm{FBS}$, respectively. All cells were maintained at $37^{\circ} \mathrm{C}$ in a humidified $5 \% \mathrm{CO}_{2}$ incubator. For hypoxic condition, cells were incubated in a hypoxic chamber (Forma Scientific, Marietta, $\mathrm{OH}$, USA) under $5 \% \mathrm{CO}_{2}$ and $1 \% \mathrm{O}_{2}$ balanced with $\mathrm{N}_{2}$.

Cell proliferation assay. Serum-starved HUVECs $\left(3 \times 10^{3}\right.$ cells/ well) seeded in a 96-well culture plate were stimulated by VEGF (30 ng/ml) with or without various concentrations of Hovenia dulcis Thunb. extract and ampelopsin for $72 \mathrm{~h}$. Cell proliferation was measured using a 3-(4,5-dimethylthiazol-2-yl)-2,5-diphenyltetrazolium bromide (MTT) colorimetric assay. Briefly, $50 \mu \mathrm{l}$ of MTT solution ( $2 \mathrm{mg} / \mathrm{ml}$; Sigma-Aldrich) was added to each well followed by incubation for $3 \mathrm{~h}$ at $37^{\circ} \mathrm{C}$. To dissolve formazan crystals, the culture medium was removed and an equal volume of DMSO was added to each well. The absorbance of each well was determined at a wavelength of $540 \mathrm{~nm}$ using a microplate reader (Thermo Scientific, Vantaa, Finland).

Chemoinvasion assay. The invasiveness of HUVECs was investigated using a Transwell chamber system with polycarbonate filter inserts with a pore size of $8.0 \mu \mathrm{m}$. Briefly, the lower side of the filter was coated with $10 \mu \mathrm{l}$ gelatin $(1 \mathrm{mg} / \mathrm{ml})$ and the upper side was coated with $10 \mu \mathrm{l}$ Matrigel $(3 \mathrm{mg} / \mathrm{ml})$. Serum-starved HUVECs $\left(8 \times 10^{4}\right.$ cells) were placed in the upper chamber of the filter and Hovenia dulcis Thunb. extract and ampelopsin were added to the lower chamber in the presence of VEGF (30 $\mathrm{ng} / \mathrm{ml})$. The chamber was incubated at $37^{\circ} \mathrm{C}$ for $18 \mathrm{~h}$, and then the cells were fixed with methanol and then stained with hematoxylin and eosin (H\&E). The total number of cells that invaded the lower chamber of the filter was counted using an optical microscope (Olympus, Center Valley, PA, USA) at a magnification of x100.

Capillary tube formation assay. Serum-starved HUVECs $\left(4 \times 10^{4}\right.$ cells) were inoculated on a surface containing Matrigel (10 mg/ml) and were incubated with Hovenia dulcis Thunb. extract and ampelopsin for $6 \mathrm{~h}$ in the presence of VEGF (30 ng/ml). Morphological changes in the cells and tube formation were visualized under a microscope and photographed at a magnification of x100 (Olympus). Tube formation was quantified by counting the total number of branched tubes in randomly selected fields at a magnification of $x 100$.

Cell migration assay. The confluent monolayer HUVECs were scratched using a tip and each well was washed with PBS to remove non-adherent cells. The cells were treated with Hovenia dulcis Thunb. extract and ampelopsin in the presence of VEGF (30 $\mathrm{ng} / \mathrm{ml}$ ) and then incubated for up to $48 \mathrm{~h}$. The perimeter of the area with a central cell-free gap was confirmed at the time intervals 0, 24, and $48 \mathrm{~h}$ under an optical microscope (Olympus).

Chorioallantoic membrane (CAM) assay. Fertilized chick eggs were maintained in a humidified incubator at $37^{\circ} \mathrm{C}$ for 3 days. Approximately 5-6 $\mathrm{ml}$ egg albumin was removed with a hypodermic needle, allowing the CAM and yolk sac to drop away from the shell membrane. After 2 days, the shell was punched out and peeled away. Thermanox coverslips (Nalge Nunc International, Rochester, NY, USA) with or without Hovenia dulcis Thunb extract and ampelopsin were air-dried and applied to the CAM surface, respectively. 
Two days later, $1 \mathrm{ml}$ of $10 \%$ intralipid (Sigma-Aldrich) was injected into the chorioallantois and the CAM was observed under a microscope.

Western blot analysis. Cell lysates were separated by $10 \%$ sodium dodecyl sulfate-polyacrylamide gel electrophoresis (SDS-PAGE), and the separated proteins were transferred to polyvinylidene difluoride (PVDF) membranes (Millipore, Billerica, MA, USA) using standard electroblotting procedures. The blots were blocked and immunolabeled with primary antibodies against phospho-VEGFR2, VEGFR2, phospho-STAT3, STAT3, phospho-AKT, AKT, phosphoERK1/2, ERK1/2, cyclin D1, MMP-2, MMP-9, HIF-1 $\alpha$ and $\beta$-actin overnight at $4^{\circ} \mathrm{C}$. Immunolabeling was detected with an enhanced chemiluminescence (ECL) kit (Bio-Rad Laboratories, Hercules, CA, USA), according to the manufacturer's instructions.

Reactive oxygen species (ROS) measurement. ROS levels were detected with 2',7'-dichlorodihydrofluorescein diacetate $\left(\mathrm{H}_{2}\right.$ DCFDA; Molecular Probes, Eugene, OR, USA). For the assay, serum-starved HUVECs seeded at a density of $1 \times 10^{5}$ cells/well in 96-black well culture plates were pretreated with Hovenia dulcis Thunb. extract and ampelopsin for $3 \mathrm{~h}$. After incubation with $\mathrm{H}_{2}$ DCFDA $(10 \mu \mathrm{M})$ for $5 \mathrm{~min}$, the cells were stimulated with VEGF $(30 \mathrm{ng} / \mathrm{ml})$ for $5 \mathrm{~min}$. The fluorescence intensity of DCF was detected using an Optinity KI-2000F fluorescence microscope (Korea Lab Tech) or a multimode microplate reader (Thermo Scientific, Vantaa, Finland) at the excitation and emission wavelengths of 495 and $529 \mathrm{~nm}$, respectively.

Measurement of VEGF by enzyme-linked immunosorbent assay (ELISA). VEGF concentration in the media from Hovenia dulcis Thunb. extract- or ampelopsin-treated cells was determined using a VEGF immunoassay kit (R\&D Systems, Minneapolis, MN, USA) according to the manufacturer's instructions. The results were expressed as the concentration of VEGF relative to the total amount of protein from each well.

Statistical analysis. The results are expressed as the mean \pm standard error (SE). Student's t-test was used to determine statistical significance between the control and the test groups. A P-value of $<0.05$ was considered to indicate a statistically significant difference.

\section{Results}

Effect of Hovenia dulcis Thunb. extract on in vitro angiogenesis. First, to determine the optimum dose of $100 \%$ ethanol extract of Hovenia dulcis Thunb. (HDT) with no cytotoxicity for angiogenesis assays, various concentrations of HDT were applied to human umbilical vein endothelial cells (HUVECs) for $72 \mathrm{~h}$. The viability of the HUVECs was not influenced up to $100 \mu \mathrm{g} / \mathrm{ml}$ of HDT treatment (Fig. 1A). Based on this result, in vitro angiogenesis assays were performed in a concentration range $(0-100 \mu \mathrm{g} / \mathrm{ml})$ of HDT at which no cytotoxicity was observed. To assess the antiangiogenic activity of HDT, we investigated the effect of HDT on the proliferation of HUVECs induced by VEGF. Serum-starved HUVECs were stimulated by VEGF with or without HDT and then cell growth was measured using the MTT colorimetric assay. As shown in Fig. 1B, HDT inhibited the VEGF-induced proliferation of HUVECs. We next examined the effect of HDT on key angiogenic phenotypes of endothelial cells such as cell invasion, tube formation and migration. HDT dosedependently decreased the VEGF-induced invasiveness, tube forming ability and migration of HUVECs at subtoxic doses (Fig. 1C-E). These results suggest that Hovenia dulcis Thunb. extract significantly inhibits VEGF-induced angiogenesis of HUVECs in vitro.

Effect of Hovenia dulcis Thunb. extract on in vivo angiogenesis. The antiangiogenic activity of HDT was also validated in vivo using a chorioallantoic membrane (CAM) assay. Coverslips containing HDT were placed on the CAM surface, and angiogenesis zones were observed under a microscope. As shown in Fig. 1F, the inhibition of neovascularization on control coverslips was $16 \%(\mathrm{n}=18)$, whereas HDT much more potently inhibited the angiogenesis of the CAM (68\% at $2 \mu \mathrm{g} / \mathrm{egg}, \mathrm{n}=16)$ without toxicity against pre-existing vessels. Taken together, these results demonstrated that Hovenia dulcis Thunb. extract efficiently inhibits angiogenesis without affecting endothelial cell viability both in vitro and in vivo.

Effect of Hovenia dulcis Thunb. extract on VEGFR2 signaling. VEGFR2 is considered the major mediator of VEGF-induced downstream signaling in endothelial cells, including survival, mitogenesis and especially angiogenesis (27-29). To examine the underlying molecular mechanism of HDT on angiogenesis, we evaluated the effect of HDT on VEGF-mediated VEGFR2 signaling pathways in HUVECs. As shown in Fig. 2A, HDT decreased VEGF-induced VEGFR2, STAT3, AKT and ERK1/2 phosphorylation in a dose-dependent manner. In addition, HDT reduced the total protein levels of VEGFR2, indicating that the inhibitory effect on VEGFR2 expression affected VEGF-induced VEGFR2 phosphorylation. VEGFR2 signaling induces the expression of endothelial cell-derived matrix metalloproteases (MMPs), including MMP-2 and MMP-9, which degrade the matrix to allow for endothelial sprouting. The cell cycle regulatory protein, cyclin D1, also plays a key role in endothelial cell proliferation during angiogenic process $(6,7)$. As shown in Fig. 2B, HDT suppressed the protein expression of MMP-2, MMP-9 and cyclin D1 in a dose-dependent manner. Furthermore, HDT remarkably reduced the VEGF-stimulated ROS generation in HUVECs (Fig. 2C). Thus, these data suggest that HDT exhibits the antiangiogenic activity by inhibiting VEGFR2-mediated downstream signaling cascades in HUVECs.

Effect of Hovenia dulcis Thunb. extract on hypoxia-induced accumulation of HIF-1 $\alpha$ protein. HIF-1 $\alpha$ is a crucial transcription factor of the angiogenesis in tumor cells, which controls transcription of hypoxia-regulated genes such as $\operatorname{VEGF}(10,11)$. Thus, we evaluated the HIF- $1 \alpha$ inhibitory activity of HDT in the human hepatoma cell line HepG2, a hypervascularized tumor. HDT-treated HepG2 cells attentively reduced the hypoxia-induced accumulation of HIF- $1 \alpha$ protein at $100 \mu \mathrm{g} / \mathrm{ml}$ (Fig. 3A). We further assessed the effect of HDT on the expression of VEGF during hypoxia. As shown in Fig. 3B, treatment 


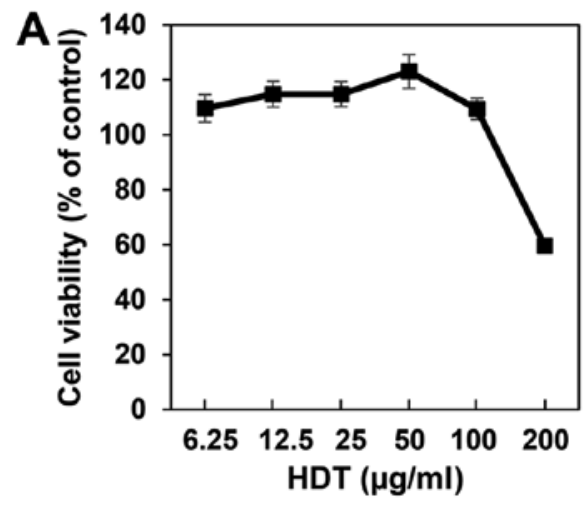

B

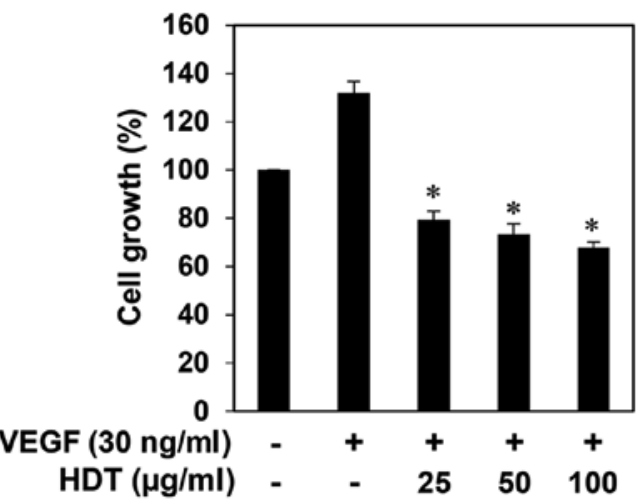

C
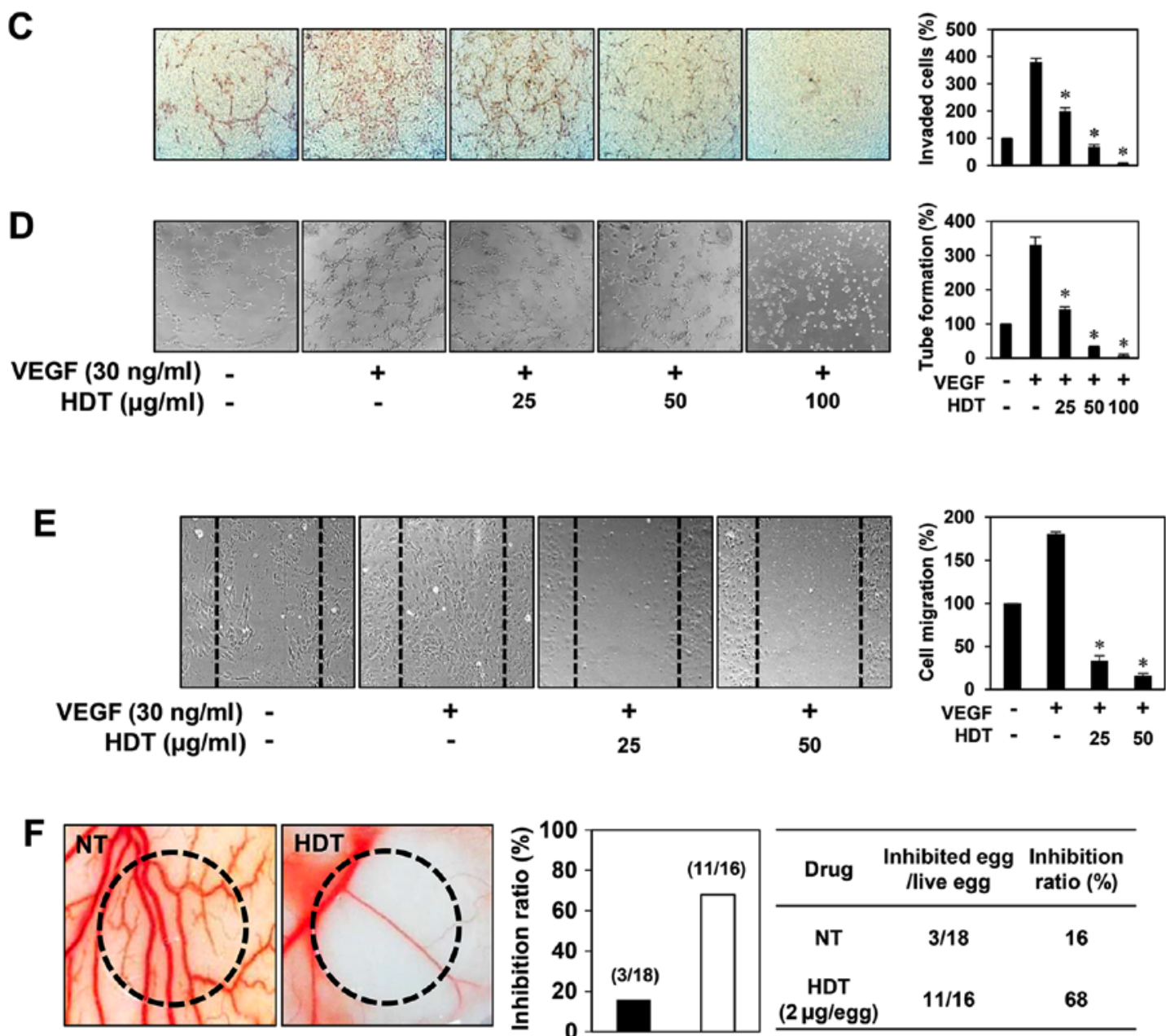

Figure 1. The in vitro and in vivo antiangiogenic activity of Hovenia dulcis Thunb. extract (HDT). (A) The effect of HDT on the viability of HUVECs. (B) The effect of HDT on the VEGF-induced growth of HUVECs. (C) The inhibitory effect of HDT on the VEGF-induced invasion of HUVECs. (D) The inhibitory effect of HDT on the VEGF-induced tube-forming ability of HUVECs. (E) The inhibitory effect of HDT on the VEGF-induced migration of HUVECs. Dotted black lines indicate the edge of the gap at $0 \mathrm{~h}$. ${ }^{*} \mathrm{P}<0.05$, compared with the VEGF control. (F) The antiangiogenic activity of HDT in chorioallantoic membranes (CAMs). Coverslips loaded with vehicle alone or HDT were applied to the CAM surface. Calculations were based on the ratio of inhibited eggs relative to the total number of live eggs.

with HDT under hypoxia decreased the VEGF production in HepG2 cells, indicating that HDT inhibits tumor angiogenesis by downregulating HIF-1 $\alpha$ and VEGF expression.

Effect of ampelopsin on in vitro and in vivo angiogenesis. Ampelopsin is a major active ingredient of Hovenia dulcis Thunb. extract (23) (Fig. 4A). The high-performance liquid chromatography (HPLC) analysis also revealed that the ethanol extract of Hovenia dulcis Thunb. branches used in the present study contained ampelopsin (Fig. 4B). Thus, we assessed whether ampelopsin is one of the possible pharmaceutically active compounds contributing to the antiangiogenic activity of Hovenia dulcis Thunb. extract. Unlike HDT, ampelopsin did not affect the VEGF-induced proliferation of HUVECs (Fig. 4C). However, ampelopsin significantly inhibited the VEGF-induced invasiveness, tube forming 

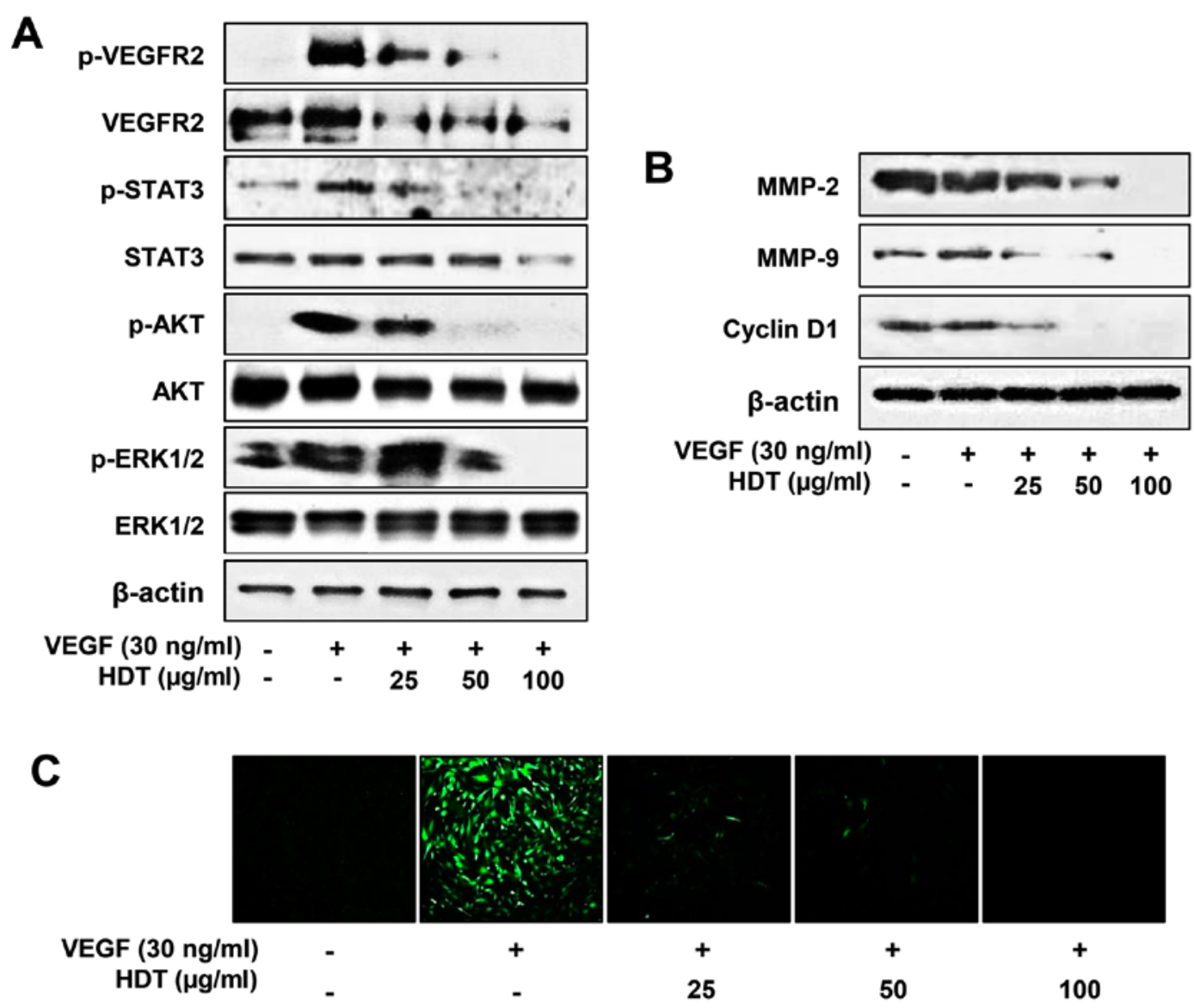

Figure 2. Downregulation of VEGFR2 signaling by Hovenia dulcis Thunb. extract (HDT). Serum-starved HUVECs were pretreated with HDT for $3 \mathrm{~h}$ at the indicated concentrations and then stimulated with VEGF (30 ng/ml) for $5 \mathrm{~min}$. (A) The effect of HDT on VEGFR2-dependent signal transduction in HUVECs. (B) The effect of HDT on MMP-2, MMP-9 and cyclin D1 protein expression in HUVECs. Protein levels were detected by western blot analysis. The level of $\beta$-actin was used as an internal control. (C) The effect of HDT on ROS generation in HUVECs. ROS levels were detected with $\mathrm{H}_{2}$ DCFDA using a fluorescence microscope.

A

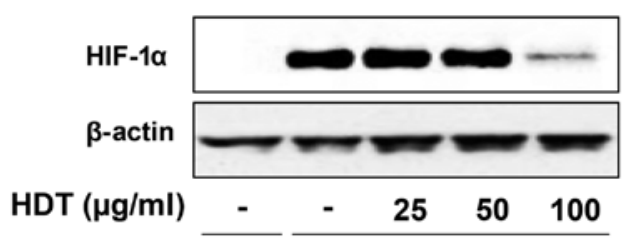

Normoxia Hypoxia

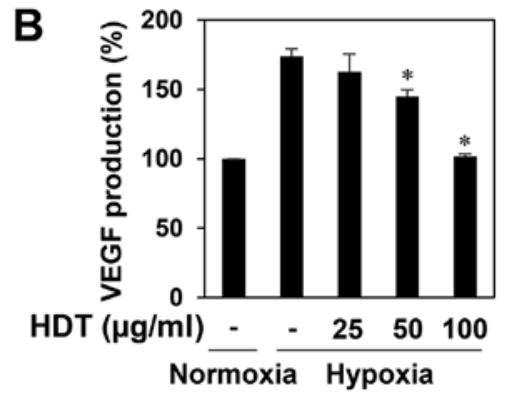

Figure 3. HIF-1 $\alpha$ inhibitory activity of Hovenia dulcis Thunb. extract (HDT). HepG2 cells were pretreated with HDT for 1 h at the indicated concentrations and then exposed to $1 \% \mathrm{O}_{2}$ for $4 \mathrm{~h}$. (A) The effect of HDT on HIF-1 $\alpha$ protein accumulation. Protein levels were measured by western blot analysis. The level of $\beta$-actin was used as an internal control. (B) The effect of HDT on VEGF expression. The concentration of VEGF protein in the culture supernatant was determined by a VEGF specific ELISA. The basal level of VEGF production in normoxia was normalized to $100 \%$. ${ }^{*} \mathrm{P}<0.05$, compared with the hypoxic control.

ability and migration of HUVECs (Fig. 4D-F). Furthermore, ampelopsin effectively inhibited the angiogenesis of the CAM ( $72 \%$ at $10 \mu \mathrm{g} / \mathrm{egg}, \mathrm{n}=18$ ) without showing any rupture of or toxicity against pre-existing vessels, when compared to the control (15\%, n=20) (Fig. 4G). These results demonstrated that ampelopsin suppresses both in vitro and in vivo angiogenesis without affecting endothelial cell proliferation.

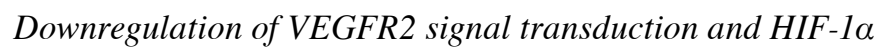
expression by ampelopsin. To elucidate whether ampelopsin affects VEGFR2 signal transduction, we investigated the effect of ampelopsin on VEGF-induced VEGFR2 signaling pathways in HUVECs. As shown in Fig. 5A, ampelopsin effectively reduced the phosphorylation of VEGFR2, AKT and ERK1/2 induced by VEGF without affecting the total protein levels. In addition, we found that ampelopsin dose-dependently reduced the generation of ROS induced by VEGF in HUVECs, indicating that the blockade of VEGFR2 signaling by ampelopsin may be associated with the downregulation of ROS generation (Fig. 5B). We next evaluated the HIF-1 $\alpha$ inhibitory activity of 
A<smiles>O=C1c2c(O)cc(O)cc2OC(c2cc(O)c(O)c(O)c2)C1O</smiles>

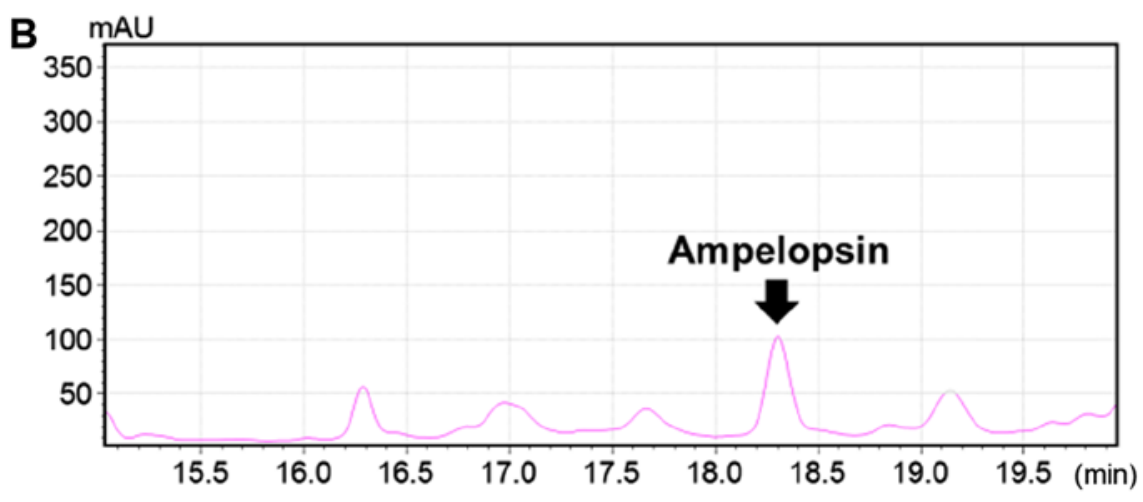

C

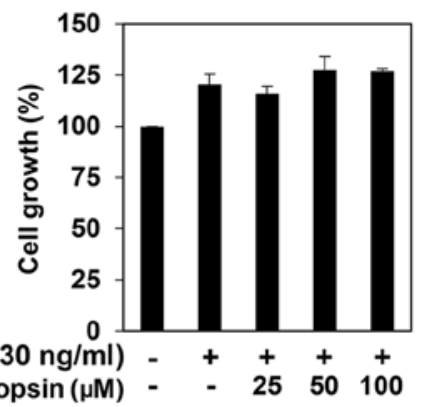

D
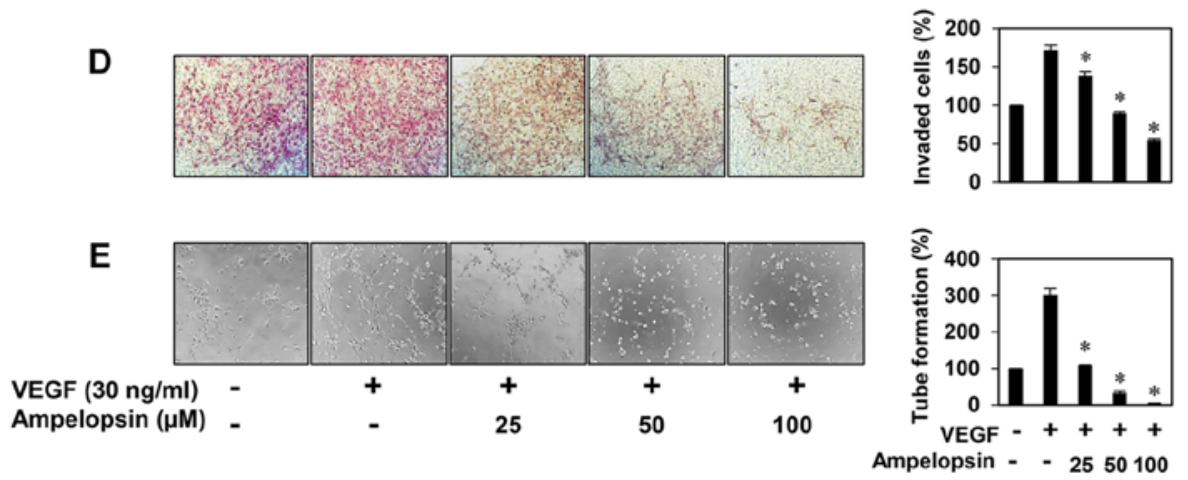

$\mathbf{F}$

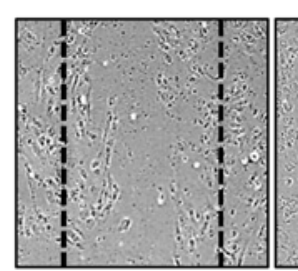

VEGF $(30 \mathrm{ng} / \mathrm{ml})$ Ampelopsin $(\mu \mathrm{M})$

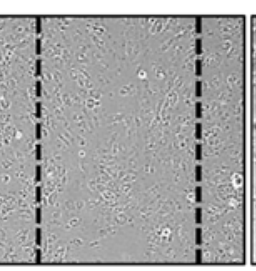

$+$

$-$

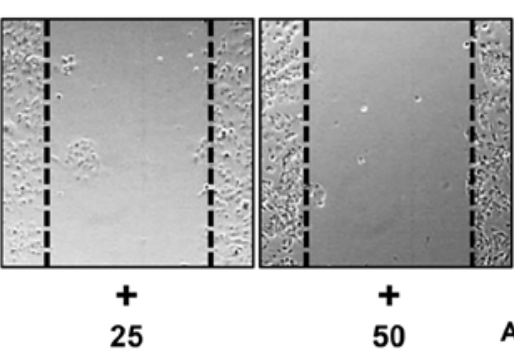

25

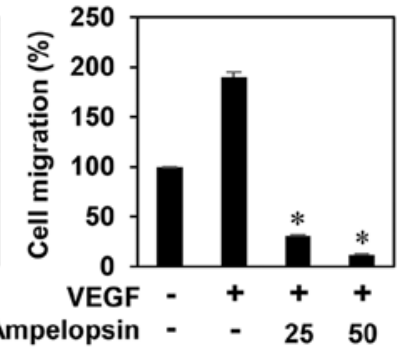

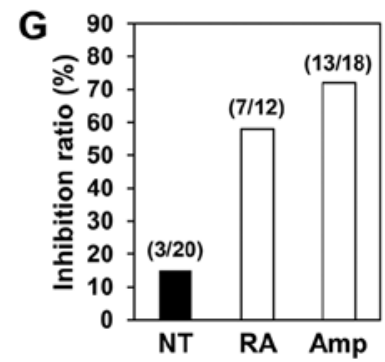

\begin{tabular}{ccc}
\hline Drug & $\begin{array}{c}\text { Inhibited egg } \\
\text { /live egg }\end{array}$ & $\begin{array}{c}\text { Inhibition } \\
\text { ratio (\%) }\end{array}$ \\
\hline NT & $3 / 20$ & 15 \\
$\begin{array}{c}\text { Retinoic acid } \\
(1 \mu \text { g/egg }) \\
\text { Ampelopsin } \\
(10 \mu g / \text { egg })\end{array}$ & $7 / 12$ & 58 \\
\hline
\end{tabular}

Figure 4. The in vitro and in vivo antiangiogenic activity of ampelopsin. (A) The chemical structure of ampelopsin. (B) HPLC chromatogram of the ethanol extract of Hovenia dulcis Thunb. branches. (C) The effect of ampelopsin on the VEGF-induced growth of HUVECs. (D) The inhibitory effect of ampelopsin on the VEGF-induced invasion of HUVECs. (E) The inhibitory effect of ampelopsin on the VEGF-induced tube forming ability of HUVECs. (F) The inhibitory effect of ampelopsin on the VEGF-induced migration of HUVECs. Dotted black lines indicate the edge of the gap at 0 h. * $\mathrm{P}<0.05$, compared with the VEGF control. (G) The antiangiogenic activity of ampelopsin in chorioallantoic membranes (CAMs). Retinoic acid (RA) was used as a positive control for anti-angiogenic responses. 
A

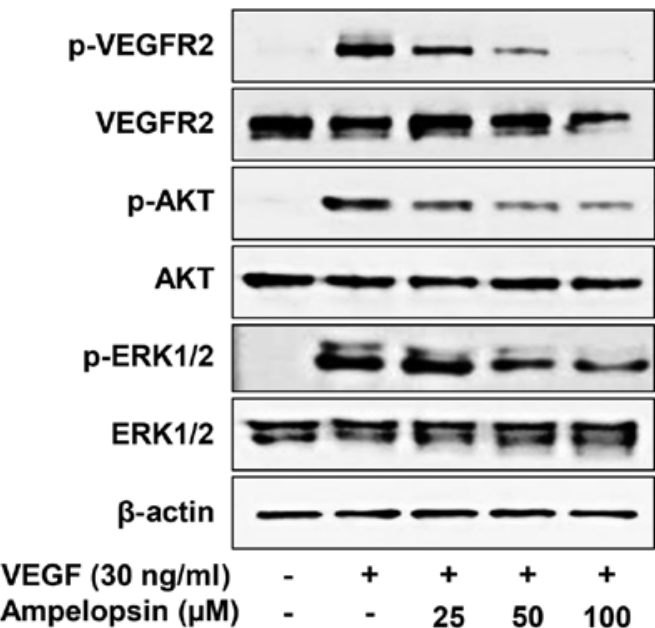

B
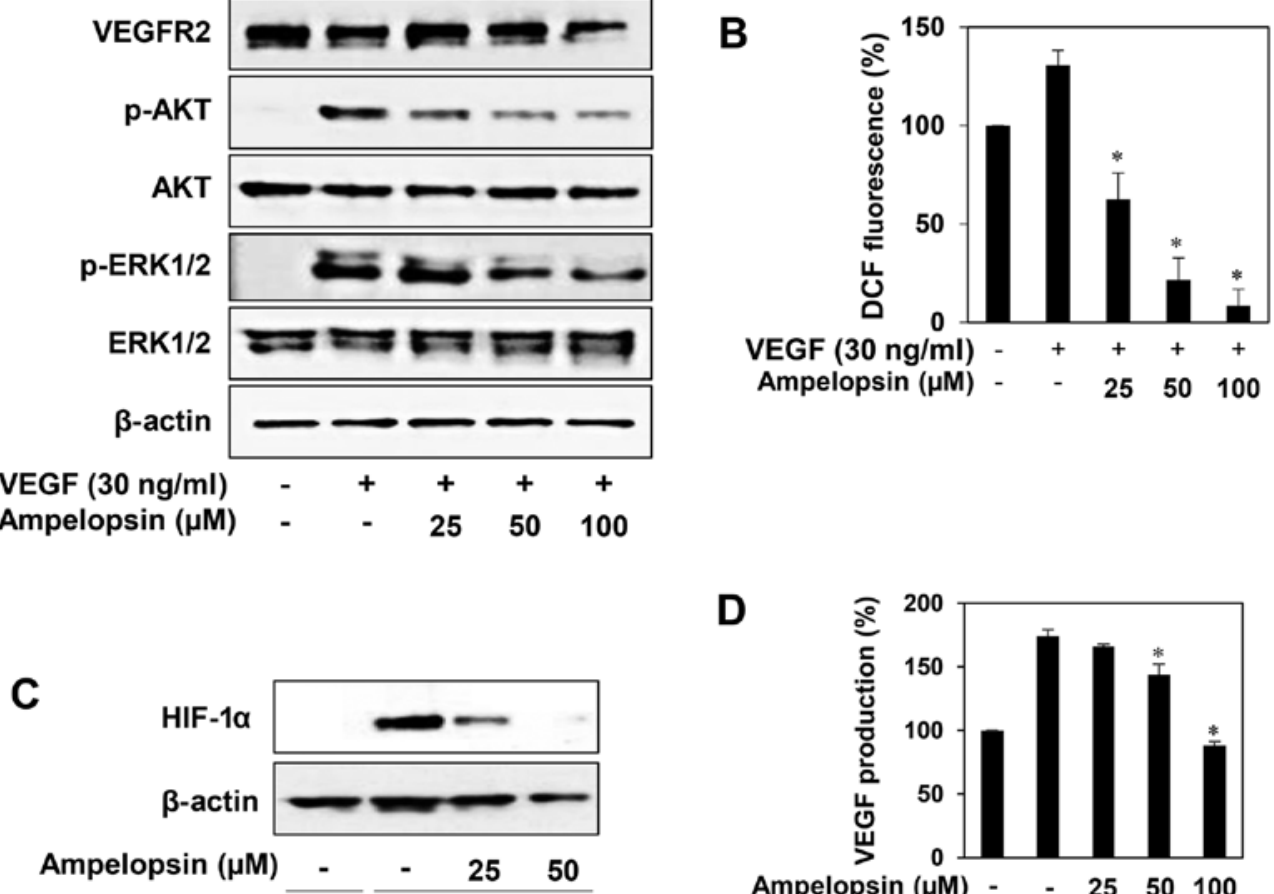

D

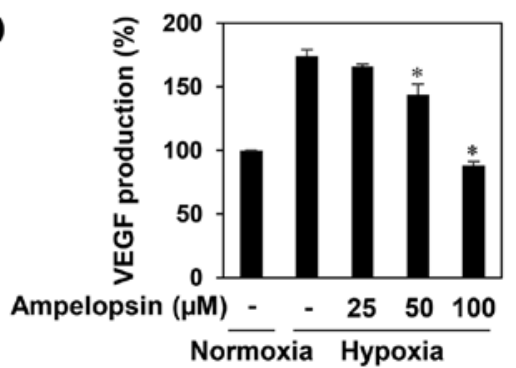

Figure 5. The antiangiogenic mechanisms of ampelopsin. (A) Downregulation of VEGFR2 signaling by ampelopsin in HUVECs. Serum-starved HUVECs were pretreated with ampelopsin for $3 \mathrm{~h}$ at the indicated concentrations and then stimulated with VEGF ( $30 \mathrm{ng} / \mathrm{ml})$ for $5 \mathrm{~min}$. (B) The effect of ampelopsin on ROS generation in HUVECs. ROS levels were detected with $\mathrm{H}_{2}$ DCFDA. "P<0.05, compared with the VEGF control. (C) The effect of ampelopsin on HIF-1 $\alpha$ protein accumulation in HepG2 cells. Cells were pretreated with ampelopsin for $1 \mathrm{~h}$ at the indicated concentrations and then exposed to $1 \% \mathrm{O}_{2}$ for $4 \mathrm{~h}$. Protein levels were measured by western blot analysis. The level of $\beta$-actin was used as an internal control. (D) The effect of ampelopsin on VEGF expression in HepG2 cells. The concentration of VEGF protein in the culture supernatant was determined by a VEGF-specific ELISA. The basal level of VEGF production in normoxia was normalized to $100 \%$. ${ }^{*} \mathrm{P}<0.05$, compared with the hypoxic control.

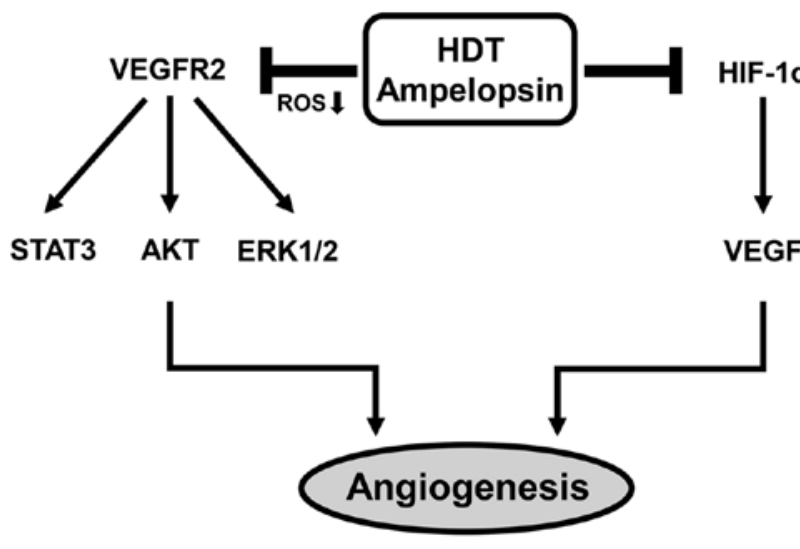

Figure 6. Role of Hovenia dulcis Thunb. extract (HDT) and ampelopsin on angiogenesis. The present study suggests that HDT and its active compound ampelopsin could suppress angiogenesis by modulating at least two angiogenic pathways, namely the blockade of VEGFR2 signal transduction and inhibition of the expression of HIF-1 $\alpha /$ VEGF.

ampelopsin in HepG2 cells. Ampelopsin prominently inhibited the expression of HIF- $1 \alpha$ and its transcriptional target, VEGF, during hypoxia (Fig. 5C and D). Taken together, these results suggest that ampelopsin shows antiangiogenic activity through the blockade of both VEGFR2 signaling and HIF-1 $\alpha$ expression and, therefore, the antiangiogenic effect of Hovenia dulcis Thunb. extract is at least in part due to ampelopsin (Fig. 6).

\section{Discussion}

To date, the discovery of angiogenesis inhibitors for the treatment of angiogenesis-related diseases including cancer has generated drug-related toxicity issues in the coagulation cascade, immune system and blood flow regulation (30). For this reason, we expect that the development of non-toxic and more potent antiangiogenic agents will lead to an improved therapeutic effect in patients as well as provide further insight into the biological process of angiogenesis. Accumulating evidence has shown that natural products play a positive role in the discovery of new bioactive compounds including antiangiogenic agents (31).

Previous studies have revealed that Hovenia dulcis Thunb. possesses various biological activities such as antifatigue, antidiabetic, neuroprotective and hepatoprotective effects (18-22). In addition, the molecular mechanisms underlying its biological effects were partially identified. Hovenia dulcis Thunb. extract reduced lipid accumulation in oleic acid-induced steatosis of $\mathrm{HepG} 2$ cells via activation of AMPK and PPAR $\alpha / \mathrm{CPT}-1$ pathway (32). Hovenia dulcis Thunb. extract also suppressed lipopolysaccharide (LPS)-stimulated inflammatory responses through nuclear factor- $\kappa \mathrm{B}$ (NF- $\kappa \mathrm{B})$ pathway in Raw 264.7 cells (33). However, the antiangiogenic activity of Hovenia dulcis Thunb. extract has not yet been reported.

Ampelopsin, a flavanonol of Hovenia dulcis Thunb. extract, possesses antiinflammatory, antioxidative and 
anticancer effects (24-26). The molecular mechanisms underlying the biological effects of ampelopsin were also partly demonstrated. Ampelopsin protected endothelial cells from hyperglycemia-induced oxidative damage by inducing autophagy via the AMPK signaling pathway (34). Ampelopsin also induced apoptosis by regulating multiple c-Myc/S-phase kinase-associated protein $2 /$ F-box and WD repeat-containing protein $7 /$ histone deacetylase 2 pathways in human lung adenocarcinoma cells (35). Moreover, ampelopsin attenuated LPS-induced inflammatory response through the inhibition of $\mathrm{NF}-\kappa \mathrm{B}$ and JAK2/STAT3 signaling pathways in microglia (24). However, whether ampelopsin inhibits angiogenesis and its underlying mechanism in endothelial cells are still unknown.

Angiogenesis, the formation of new blood vessels, proceeds by a multistep process, including endothelial cell proliferation, migration, tube formation and invasion. Inhibition at any step may result in suppression of angiogenesis (36). Our results importantly showed that Hovenia dulcis Thunb. extract dose-dependently inhibited VEGF-induced endothelial cell proliferation, whereas ampelopsin had no affect. Thus, the antiproliferative effect of Hovenia dulcis Thunb. extract may be associated with the action of another bioactive component in the extract other than ampelopsin. Nevertheless, our results demonstrated that Hovenia dulcis Thunb. extract and ampelopsin exhibited potent inhibitory effects on VEGFinduced invasion, tube formation and migration of HUVECs. Furthermore, our data showed that Hovenia dulcis Thunb. extract and ampelopsin inhibited in vivo neovascularization of the CAM without toxicity.

VEGFR2 signaling is a main pathway involved in angiogenesis. Therefore, suppression of the VEGFR2 signal transduction cascade is the major strategy in antiangiogenic therapy. In addition, targeting the transcription regulators of proangiogenic factors such as HIF-1 $\alpha$ in cancer cells can be a powerful strategy to effectively inhibit tumor angiogenesis through prevention of VEGF expression $(37,38)$. In this study, we demonstrated that Hovenia dulcis Thunb. extract and ampelopsin downregulated both VEGFR2 signaling of endothelial cells and the HIF-1 $\alpha$ expression of tumor cells. Notably, unlike ampelopsin, Hovenia dulcis Thunb. extract also reduced the total protein levels of VEGFR2, indicating that other ingredients of the extract may result in the inhibitory effect on VEGFR2 expression. Meanwhile, Hovenia dulcis Thunb. extract and ampelopsin suppressed the VEGF-induced generation of ROS in HUVECs. In recent studies, VEGF stimulated ROS production and in turn promoted VEGFR2 autophosphorylation by reversibly oxidizing and inactivating protein tyrosine phosphatases (PTPs) $(39,40)$. Therefore, they may block the VEGFR2 signaling via the downregulation of ROS generation. In addition, ampelopsin more potently inhibited the expression of HIF-1 $\alpha$ in HepG2 hepatoma cells than Hovenia dulcis Thunb. extract. Thus, ampelopsin could be a promising antiangiogenic agent for cancer treatment.

In conclusion, our results demonstrated that the antiangiogenic effects of Hovenia dulcis Thunb. extract and its active compound ampelopsin on HUVECs were exerted through the inhibition of VEGFR2 signal transduction, leading to decreased cell proliferation, migration, invasion and tube formation. Hovenia dulcis Thunb. extract and ampelopsin may also exert inhibitory activity on tumor angiogenesis by down- regulating the expression of HIF-1 $\alpha$. In addition, we found that Hovenia dulcis Thunb. extract and ampelopsin could suppress basic fibroblast growth factor (bFGF)-stimulated invasion and tube formation of HUVECs in vitro, suggesting that they may also affect other growth factor-related angiogenesis (data not shown). These findings provide support for the potential use of Hovenia dulcis Thunb. extract and ampelopsin for antiangiogenic therapy. However, further investigation of their therapeutic effects in angiogenesis-related disease models and action mechanisms would be required for their development as natural pharmaceutical agents.

\section{Acknowledgements}

This study was carried out with the support of the 'Cooperative Research Program for Agriculture Science and Technology Development (project no. PJ01188001)' Rural Development Administration, Republic of Korea, the Basic Science Research Program through the National Research Foundation of Korea (NRF) funded by the Ministry of Education (NRF-2016R1D1A1B03932956), and the Brain Korea 21 Plus Project, Republic of Korea.

\section{References}

1. Folkman J: Seminars in Medicine of the Beth Israel Hospital, Boston. Clinical applications of research on angiogenesis. NEngl J Med 333: 1757-1763, 1995.

2. Risau W: Mechanisms of angiogenesis. Nature 386: 671-674, 1997.

3. Karamysheva AF: Mechanisms of angiogenesis. Biochemistry (Mosc) 73: 751-762, 2008.

4. Holash J, Wiegand SJ and Yancopoulos GD: New model of tumor angiogenesis: Dynamic balance between vessel regression and growth mediated by angiopoietins and VEGF. Oncogene 18: 5356-5362, 1999.

5. Holmes K, Roberts OL, Thomas AM and Cross MJ: Vascular endothelial growth factor receptor-2: Structure, function, intracellular signalling and therapeutic inhibition. Cell Signal 19: 2003-2012, 2007.

6. Stetler-Stevenson WG: Matrix metalloproteinases in angiogenesis: A moving target for therapeutic intervention. J Clin Invest 103: 1237-1241, 1999.

7. Baldin V, Lukas J, Marcote MJ, Pagano M and Draetta G: Cyclin D1 is a nuclear protein required for cell cycle progression in G1. Genes Dev 7: 812-821, 1993.

8. Semenza GL: Regulation of mammalian $\mathrm{O}_{2}$ homeostasis by hypoxia-inducible factor 1 . Annu Rev Cell Dev Biol 15: 551-578, 1999.

9. Höckel M and Vaupel P: Tumor hypoxia: Definitions and current clinical, biologic, and molecular aspects. J Natl Cancer Inst 93: 266-276, 2001.

10. Greer SN, Metcalf JL, Wang Y and Ohh M: The updated biology of hypoxia-inducible factor. EMBO J 31: 2448-2460, 2012.

11. Forsythe JA, Jiang BH, Iyer NV, Agani F, Leung SW, Koos RD and Semenza GL: Activation of vascular endothelial growth factor gene transcription by hypoxia-inducible factor 1 . Mol Cell Biol 16: 4604-4613, 1996.

12. Fan TP, Yeh JC, Leung KW, Yue PY and Wong RN: Angiogenesis: From plants to blood vessels. Trends Pharmacol Sci 27: 297-309, 2006.

13. Avramis IA, Kwock R and Avramis VI: Taxotere and vincristine inhibit the secretion of the angiogenesis inducing vascular endothelial growth factor (VEGF) by wild-type and drug-resistant human leukemia T-cell lines. Anticancer Res 21: 2281-2286, 2001.

14. Clements MK, Jones CB, Cumming M and Daoud SS: Antiangiogenic potential of camptothecin and topotecan. Cancer Chemother Pharmacol 44: 411-416, 1999.

15. Vincent L, Kermani P, Young LM, Cheng J, Zhang F, Shido K, Lam G, Bompais-Vincent H, Zhu Z, Hicklin DJ, et al: Combretastatin A4 phosphate induces rapid regression of tumor neovessels and growth through interference with vascular endothelial-cadherin signaling. J Clin Invest 115: 2992-3006, 2005. 
16. Lee JH, Choi S, Lee Y, Lee HJ, Kim KH, Ahn KS, Bae H, Lee HJ, Lee EO, Ahn KS, et al: Herbal compound farnesiferol C exerts antiangiogenic and antitumor activity and targets multiple aspects of VEGFR1 (Flt1) or VEGFR2 (Flk1) signaling cascades. Mol Cancer Ther 9: 389-399, 2010.

17. Han JM, Kwon HJ and Jung HJ: Tricin, 4',5,7-trihydroxy-3',5'dimethoxyflavone, exhibits potent antiangiogenic activity in vitro. Int J Oncol 49: 1497-1504, 2016.

18. Yang J, Wu S and Li C: High efficiency secondary somatic embryogenesis in Hovenia dulcis Thunb. through solid and liquid cultures. Sci World J 2013: 718754, 2013.

19. Na CS, Yoon SY, Kim JB, Na DS, Dong MS, Lee MY and Hong CY: Anti-fatigue activity of Hovenia dulcis on a swimming mouse model through the inhibition of stress hormone expression and antioxidation. Am J Chin Med 41: 945-955, 2013.

20. Ji Y, Chen S, Zhang K and Wang W: Effects of Hovenia dulcis Thunb on blood sugar and hepatic glycogen in diabetic mice. Zhong Yao Cai 25: 190-191, 2002 (In Chinese).

21. Li G, Min BS, Zheng C, Lee J, Oh SR, Ahn KS and Lee HK: Neuroprotective and free radical scavenging activities of phenolic compounds from Hovenia dulcis. Arch Pharm Res 28 804-809, 2005

22. Hase K, Ohsugi M, Xiong Q, Basnet P, Kadota S and Namba T: Hepatoprotective effect of Hovenia dulcis THUNB. on experimental liver injuries induced by carbon tetrachloride or D-galactosamine/ lipopolysaccharide. Biol Pharm Bull 20: 381-385, 1997.

23. Park JS, Kim IS, Shaheed Ur Rehman, Na CS and Yoo HH: HPLC determination of bioactive flavonoids in Hovenia dulcis fruit extracts. J Chromatogr Sci 54: 130-135, 2016.

24. Weng L, Zhang H, Li X, Zhan H, Chen F, Han L, Xu Y and Cao X: Ampelopsin attenuates lipopolysaccharide-induced inflammatory response through the inhibition of the NF- $\mathrm{KB}$ and JAK2/ STAT3 signaling pathways in microglia. Int Immunopharmacol 44: $1-8,2017$

25. Hou X, Zhang J, Ahmad H, Zhang H, Xu Zand Wang T: Evaluation of antioxidant activities of ampelopsin and its protective effect in lipopolysaccharide-induced oxidative stress piglets. PLoS One 9: e108314, 2014.

26. Zhou Y, Liang X, Chang H, Shu F, Wu Y, Zhang T, Fu Y, Zhang Q, Zhu JD and Mi M: Ampelopsin-induced autophagy protects breast cancer cells from apoptosis through Akt-mTOR pathway via endoplasmic reticulum stress. Cancer Sci 105: $1279-1287,2014$.

27. Parast CV, Mroczkowski B, Pinko C, Misialek S, Khambatta G and Appelt K: Characterization and kinetic mechanism of catalytic domain of human vascular endothelial growth factor receptor-2 tyrosine kinase (VEGFR2 TK), a key enzyme in angiogenesis. Biochemistry 37: 16788-16801, 1998.
28. Hicklin DJ and Ellis LM: Role of the vascular endothelial growth factor pathway in tumor growth and angiogenesis. J Clin Oncol 23: 1011-1027, 2005.

29. Olsson AK, Dimberg A, Kreuger J and Claesson-Welsh L: VEGF receptor signalling - in control of vascular function. Nat Rev Mol Cell Biol 7: 359-371, 2006.

30. Verheul HM and Pinedo HM: Possible molecular mechanisms involved in the toxicity of angiogenesis inhibition. Nat Rev Cancer 7: 475-485, 2007.

31. Newman DJ and Cragg GM: Natural products as sources of new drugs over the last 25 years. J Nat Prod 70: 461-477, 2007.

32. Kim B, Woo MJ, Park CS, Lee SH, Kim JS, Kim B, An S and Kim SH: Hovenia dulcis extract reduces lipid accumulation in oleic acid-induced steatosis of Hep G2 cells via activation of AMPK and PPAR $\alpha / C P T-1$ pathway and in acute hyperlipidemia mouse model. Phytother Res 31: 132-139, 2017.

33. Park JY, Moon JY, Park SD, Park WH, Kim H and Kim JE: Fruits extracts of Hovenia dulcis Thunb. suppresses lipopolysaccharide-stimulated inflammatory responses through nuclear factor-kappaB pathway in Raw 264.7 cells. Asian Pac J Trop Med 9: 357-365, 2016

34. Liang X, Zhang T, Shi L, Kang C, Wan J,Zhou Y,Zhu J and Mi M: Ampelopsin protects endothelial cells from hyperglycemiainduced oxidative damage by inducing autophagy via the AMPK signaling pathway. Biofactors 41: 463-475, 2015.

35. Chen XM, Xie XB, Zhao Q, Wang F, Bai Y, Yin JQ, Jiang H, Xie XL, Jia Q and Huang G: Ampelopsin induces apoptosis by regulating multiple $\mathrm{c}-\mathrm{Myc} / \mathrm{S}$-phase kinase-associated protein $2 / \mathrm{F}$-box and WD repeat-containing protein $7 /$ histone deacetylase 2 pathways in human lung adenocarcinoma cells. Mol Med Rep 11: 105-112, 2015.

36. Cho SG, Yi Z, Pang X, Yi T, Wang Y, Luo J, Wu Z, Li D and Liu M: Kisspeptin-10, a KISS1-derived decapeptide, inhibits tumor angiogenesis by suppressing Sp1-mediated VEGF expression and FAK/Rho GTPase activation. Cancer Res 69: 7062-7070, 2009

37. El-Kenawi AE and El-Remessy AB: Angiogenesis inhibitors in cancer therapy: Mechanistic perspective on classification and treatment rationales. Br J Pharmacol 170: 712-729, 2013.

38. Chen SH, Murphy DA, Lassoued W, Thurston G, Feldman MD and Lee WM: Activated STAT3 is a mediator and biomarker of VEGF endothelial activation. Cancer Biol Ther 7: 1994-2003, 2008.

39. Jung HJ, Kim Y, Chang J, Kang SW, Kim JH and Kwon HJ: Mitochondrial UQCRB regulates VEGFR2 signaling in endothelial cells. J Mol Med (Berl) 91: 1117-1128, 2013.

40. Ushio-Fukai M: VEGF signaling through NADPH oxidasederived ROS. Antioxid Redox Signal 9: 731-739, 2007. 